\title{
EXISTENCE AND REGULARITY RESULTS FOR MAXWELL'S EQUATIONS IN THE QUASI-STATIC LIMIT
}

\author{
A. L. CAREY ${ }^{1}$ AND D. M. O'BRIEN ${ }^{2}$
}

(Received 5 December 1984; revised 29 July 1985)

\begin{abstract}
We prove the existence of solutions of Maxwell's equations for a conducting medium whose constitutive parameters are piecewise constant on $\mathbf{R}^{3}$, and then examine the convergence of these solutions in the quasi-static limit in which displacement currents are neglected. Secondly, we examine the regularity of the limiting solution and the sense in which the classical boundary conditions hold, namely, continuity of the tangential electric field and the normal current density.
\end{abstract}

\section{Introduction}

The work described in this paper arose from a study of the electromagnetic prospecting technique used by geophysicists. The aim of the technique is to determine the extent and electrical properties of a conducting ore body, buried in a layered conducting ground, from transient electromagnetic observations at the surface. In the usual experimental arrangement, a horizontal transmitting loop is laid upon the ground and is driven by a current pulse or current ramp. The fields

\footnotetext{
${ }^{1}$ Department of Mathematics, Research School of Physical Sciences, The Australian National University, P.O. Box 4, Canberra 2601.

${ }^{2}$ CSIRO, Division of Atmospheric Research, Private Bag No. 1, Mordialloc, Victoria.

(C) Copyright Australian Mathematical Society 1986, Serial-fee code 0334-2700/86
} 
induced in the buried ore body then generate secondary currents in a receiving loop, and these are recorded for subsequent analysis. In order to model this technique, one must be able to solve Maxwell's equations in a structured medium. When the structure is simple, such as in a layered medium without ore body, or a spherical or cylindrical ore body in a non-conducting earth, then the techniques of classical analysis yield solutions in series of special functions which are easily computable and fairly reliable. In more complex structures, one is forced to use a finite element or finite difference representation of the electromagnetic fields. In addition, it is sometimes necessary to couple the finite element representation to an integral representation in order to adequately account for boundary conditions at infinity. The successful application of finite elements and subsequent analysis of the errors presumes knowledge of the regularity of the electromagnetic fields near the interfaces. According to classical electromagnetism the tangential component of both the electric and magnetic field intensities must be continuous across any interface between media with different constitutive parameters. However, greater precision is needed in order to tackle the numerical analysis of Maxwell's equations. In particular, in what sense must the tangential components be continuous, and how discontinuous are the normal components? We were unable to find definitive answers in the literature for Maxwell's equations in a complex medium. Nor could we find results immediately applicable in the quasi-static limit, used in the electromagnetic prospecting problem in which Maxwell's equations degenerate into a parabolic system. This paper is an attempt to answer these questions. As such it is a first step in extending the approach of [3] (for a layered earth) to more complicated geometries.

In this paper we are concerned with existence and regularity results for Maxwell's equations in a conducting medium,

$$
\left.\begin{array}{c}
\nabla \times E=-\mu \partial H / \partial t \\
\nabla \times H=\varepsilon \partial E / \partial t+J \\
\nabla \cdot J=-\partial \rho / \partial t \\
J=\sigma E+K
\end{array}\right\}
$$

in the quasi-static limit as $\varepsilon \rightarrow 0$. The notation used here is standard:

(1) $E$ and $H$ are the electric and magnetic field intensities;

(2) $\rho$ is the density of electric charge;

(3) $J$ is the total current density, $\sigma E$ is the conduction current density, and $K$ is a known current density maintained by an external energy source;

(4) $\mu$ is the permeability, $\varepsilon$ the permittivity and $\sigma$ the conductivity.

We shall require that $\mu$ be constant on $\mathbf{R}^{3}$, but allow $\varepsilon$ and $\sigma$ to be piecewise constant functions. More precisely, we suppose that $\Omega_{1}, \Omega_{2}, \ldots, \Omega_{n}$ are disjoint 
open regions whose closures cover $\mathbf{R}^{3}$ and whose boundaries are smooth and that

$$
\left.\begin{array}{c}
\varepsilon(x)=\varepsilon_{i} \\
\sigma(x)=\sigma_{i}
\end{array}\right\}, \quad x \in \Omega_{t},
$$

In addition, we shall suppose that the source $K$ is switched on at time zero, and that $E, H$ and $\rho$ are all zero prior to time zero. Consequently, $\rho$ will vanish for all time in the interior of regions where $\varepsilon / \sigma$ is constant, except possibly at points where $\nabla \cdot K$ is non-zero, as the charge density will be a distribution concentrated on the interfaces between regions with different values of $\varepsilon / \sigma$.

In physical terms, the quasi-static limit is equivalent to the neglect of displacement currents. This is usually a good approximation in the propagation of low-frequency waves through conducting media, and also in the transient electromagnetic (TEM) response of a conducting medium when observations are made long after the passage of the wave fronts. One practical application, already mentioned, is to TEM prospecting, in which geophysicists measure the response of the conducting earth to a controlled current source in the search for buried ore bodies and oil deposits. This application has an extensive literature, which can be traced from the text by Wait [4].

Duvaut and Lions [1] have given an elegant existence proof for the case in which $\varepsilon$ is non-zero, but their technique fails in the quasi-static limit. Our approach is to deal directly with the equation for $E$, namely

$$
\mu \varepsilon \partial^{2} E / \partial t^{2}+\mu \sigma \partial E / \partial t+\nabla \times(\nabla \times E)=-\mu \partial K / \partial t
$$

which holds under our assumptions that $\mu$ is constant. We apply the Laplace transform to equation (2) and solve the resulting elliptic problem,

$$
\left(\mu \varepsilon s^{2}+\mu \sigma s+\nabla \times \nabla \times\right) e=-\mu s k
$$

where the lower-case $e$ and $k$ denote the Laplace transforms of $E$ and $K$, and $s$ is the Laplace transform variable. The solution of (3) satisfies a bound

$$
\|e\| \leqslant 2 \mu\left(\varepsilon_{*} \text { real } s+\sigma_{*}\right)^{-1}\|k\|,
$$

where $\varepsilon_{*}$ and $\sigma_{*}$ denote the minima of $\varepsilon$ and $\sigma$. This bound is well behaved as $\varepsilon_{*} \rightarrow 0$, and so enables us to pass to the quasi-static limit.

An open and interesting question is what happens to the results if $\sigma_{*}$ is zero (with $\sigma$ not identically zero)? Explicit calculations for the special case of a half space (ie. $\sigma(x)=0, x_{3}>0 ; \sigma(x)=$ constant $x_{3} \leqslant 0$ ) show that the solution is not $L^{2}$, so that one expects solutions in weighted Sobolev spaces in general.

The classical boundary conditions are that the tangential components of $e$ and the normal component of $\sigma e$ should be continuous across any section, $\Gamma$, of the interface between two regions, $\Omega_{+}$and $\Omega_{-}$, with different constitutive parameters. 
We use elliptic regularity to establish differentiability of $e$ away from an interface and then in order to analyse the boundary conditions, we resolve $e$ into its transverse component $e_{T}$ and longitudinal component $e_{L}$,

$$
e=e_{T}+e_{L} \text {. }
$$

We then show that $e_{T}$ lies in the Sobolev space $H^{2}$, which implies that all components of $e_{T}$ are continuous. Next we show that $n \times e_{L}$, where $n$ is the normal to $\Gamma$, is continuous in the sense that the traces of $n \times e_{L}$ on $\Gamma$ from $\Omega_{+}$ and $\Omega_{-}$are equal as distributions in $H^{-1 / 2}(\Gamma)$. Together, these results establish the (weak) continuity of $n \times e$ across $\Gamma$. Lastly, we show that $n .(\sigma e)$ is continuous across $\Gamma$ in a similar sense.

Whether stronger continuity results are possible we leave as an open question.

We do not distinguish notationally between scalar and vector fields on $\mathbf{R}^{3}$, nor between spaces of such fields. Thus, $H^{r}$ will denote the usual Sobolev space of $r$ times weakly differentiable functions on $\mathbf{R}^{3}$ with the inner product

$$
(u, v)_{r}=\sum_{|\alpha| \leqslant r} \int_{\mathbf{R}^{3}} \overline{D^{\alpha} u} D^{\alpha} v
$$

and corresponding norm

$$
\|u\|_{r}=(u, u)_{r}^{1 / 2},
$$

as well as the similar space of vector functions on $\mathbf{R}^{3}$ with inner product

$$
(u, v)_{r}=\sum_{|\alpha| \leqslant r} \int_{\mathbf{R}^{3}} \sum_{i=1}^{3} \overline{D^{\alpha} u_{i}} D^{\alpha} v_{\imath}
$$

and corresponding norm

$$
\|u\|_{r}=(u, u)_{r}^{1 / 2} \text {. }
$$

When $r=0$, we will omit the subscript from both the norm and inner product. All other notation is standard and follows, for example, that in reference [1].

\section{Existence}

Our proof of the existence theorem follows the traditional approach in which the initial-value problem for Maxwell's equations is converted into an elliptic equation, after Laplace transformation of the time coordinate. In order to be able to guarantee that the Laplace transform can be inverted, we have placed fairly strong smoothness conditions on the source of the electromagnetic fields. In fact, these conditions are too strong for the most commonly used model of the source current, namely, a square current pulse. However, the conditions can be weakened 
but only at the expense of introducing distributional Laplace transforms, with attendant notational complications, which we felt unwarranted for this paper. A second feature of the proof is the division of the fields into longitudinal and transverse components. The significance of this procedure is that the longitudinal and transverse components have different regularity properties.

We begin with the classical result of Helmholtz, which asserts that any vector field can be decomposed uniquely into longitudinal and transverse fields. Let $P_{T}$ and $P_{L}$ denote the projections on $L^{2}$ defined by:

$$
P_{T}+P_{L}=1, \quad\left(\widehat{P_{L} u}\right)_{i}(P)=\sum_{j=1}^{3}\left(p_{i} p_{j} / p^{2}\right) \hat{u}_{j}(p), \quad i=1,2,3,
$$

where $\wedge$ denotes the Fourier transform. Then in fact $P_{L}$ is an orthogonal projection on $H^{r}$ for any $r \geqslant 0$. To see this, note that

$$
\begin{aligned}
\int\left(1+|p|^{2}\right)^{r}\left|u_{L}\right|^{2} d^{3} p & =\int\left(1+|p|^{2}\right)^{r}|p \cdot u|^{2} / p^{2} d^{3} p \\
& \leqslant \int\left(1+|p|^{2}\right)^{r}|u|^{2} d^{3} p,
\end{aligned}
$$

and so if $u$ is in $H^{r}$, then the right-hand side is finite, which establishes that $u_{L}$ also lies in $H^{r}$. For any $u \in H^{r}$, we let

$$
u_{T}=P_{T} u \text { and } u_{L}=P_{L} u,
$$

and call $u_{T}$ and $u_{L}$ the transverse and longitudinal components of $u$. It is easy to check that these components satisfy

$$
\nabla \times u_{L}=0 \text { and } \nabla \cdot u_{T}=0 .
$$

In order to formulate the existence and regularity results we need some technicalities. The first of these we record as

LEMMA 1. $u \in H^{r+1}$ if and only if $u \in H^{r}, \nabla \times u \in H^{r}$ and $\nabla \cdot u \in H^{r}$, $r \geqslant 0$.

To prove this, use the identity

$$
|p \times u|^{2}=p^{2}|u|^{2}-|p \cdot u|^{2}
$$

to give

$$
\int\left(1+p^{2}\right)^{r+1}|u|^{2}=\int\left(1+|p|^{2}\right)^{r}\left[|u|^{2}+|p \times u|^{2}+|p \cdot u|^{2}\right] .
$$

Consequently, $u \in H^{r+1}$ if and only if all terms on the right are finite, that is, $u \in H^{r}, \nabla \times u \in H^{r}$ and $\nabla \cdot u \in H^{r}$. 
We now turn to the Laplace-transformed version of the operator in equation (2), namely,

$$
q+A
$$

where

$$
q=\mu \varepsilon s^{2}+\mu \sigma s,
$$

and $A$ is the operator defined as follows. Let

$$
D(A)=\left\{u \mid u \in L^{2}, \nabla \times u \in L^{2} \text { and } \nabla \times(\nabla \times u) \in L^{2}\right\},
$$

and let $A$ denote the operator on $L^{2}$ with domain $D(A)$ and action

$$
A u=\nabla \times(\nabla \times u), \quad u \in D(A) .
$$

Note that Lemma 1 shows that $D(A)$ has an alternative characterisation:

$$
D(A)=\left\{u \mid u \in L^{2}, \nabla \times u \in H^{1}\right\} .
$$

We shall show that the equation

$$
(q+A) u=f
$$

has a unique solution $u$ in $D(A)$ for any $f$ in $L^{2}$. From this solution we shall construct the solution of Maxwell's equations by inverse Laplace transformation. To do this we need a second technical result, namely that $A$ is non-negative and self-adjoint. The former is easy, since for any $u \in D(A)$,

$$
(u, A u)=\int|\nabla \times u|^{2} \geqslant 0
$$

so $A$ is non-negative.

We prove next that $A$ is closed. Suppose that $\left\{u_{i}\right\}$ is a sequence in $D(A)$ which converges to $u$ in $L^{2}$ and that $\left\{A u_{1}\right\}$ converges to $v$ in $L^{2}$. Because $\nabla \times(\nabla \times$ $\left.u_{1}\right) \rightarrow \nabla \times(\nabla \times u)$ in $D^{\prime}$, and because $v \in L^{2}$, we see that $\nabla \times(\nabla \times u) \in L^{2}$. Consequently, $u \in D(A)$ and $A u=v$. Hence $A$ is closed.

Now let $u_{*} \in D\left(A^{*}\right)$. Then the form $u \rightarrow\left(A u, u_{*}\right)$ is certainly continuous for all $u \in D$. For such $u$,

$$
\begin{aligned}
\left(A u, u_{*}\right) & =\int \overline{\nabla \times(\nabla \times u)} \cdot u_{*} \\
& =\int \bar{u} \cdot \nabla \times\left(\nabla \times u_{*}\right) .
\end{aligned}
$$

Thus, $\nabla \times\left(\nabla \times u_{*}\right) \in L^{2}$ from which it follows that $A u_{*} \in L^{2}$ and $u_{*} \in D(A)$. Since $A$ is symmetric, this establishes that $D(A)=D\left(A^{*}\right)$, and hence that $A$ is self-adjoint.

We may now state the main result of this section.

THEOREM. Let

$$
s=\alpha+i \beta,
$$


and require that $\alpha>0$. The equation

$$
(q+A) u=f
$$

has a unique solution for any $f$ in $L^{2}$. If $\varepsilon_{*}$ and $\sigma_{*}$ denote the minima of $\varepsilon$ and $\sigma$, then

$$
\|u\| \leqslant 2|s|^{-1}\left(\varepsilon_{*} \alpha+\sigma_{*}\right)^{-1}\|f\| .
$$

Lastly, if $f$ is holomorphic in $s$ in the region $\alpha>\Theta \geqslant 0$, then so too is $u$.

Proof. The proof uses a Galerkin approximation and relies on the positivity of $A, \varepsilon$ and $\sigma$. Let $v_{1}, v_{2}, \ldots$ be a basis for $D(A)$ and suppose that

$$
u=\sum_{i=1}^{m} c_{i} v_{t}
$$

where $c_{1}, c_{2}, \ldots, c_{m}$ are chosen so that

$$
\sum_{i=1}^{m} c_{i}\left(v_{j},(q+A) v_{i}\right)=\left(v_{j}, f\right)
$$

Note, firstly that $\operatorname{det}\left(v_{j},(q+A) v_{i}\right) \neq 0$. Indeed, if this were not so, there would exist $c_{1}, c_{2}, \ldots, c_{m}$ such that

$$
\sum_{i=1}^{m} c_{i}\left(v_{\jmath},(q+A) v_{i}\right)=0
$$

and hence that

$$
(u,(q+A) u)=0 .
$$

Since $A$ is positive, equation (7) implies that

$$
(u, \varepsilon u)\left(\alpha^{2}-\beta^{2}\right)+(u, \sigma u) \alpha \leqslant 0
$$

and

$$
(u, \varepsilon u) 2 \alpha \beta+(u, \sigma u) \beta=0
$$

The only possible solution of (9) is $\beta=0$, since $\varepsilon \geqslant 0, \sigma>0$ and $\alpha>0$, which is inconsistent with (8). Returning to equation (6), we find that

$$
\sum_{t, j=1}^{m} c_{\imath} \bar{c}_{j}\left(v_{j},(q+A) v_{\imath}\right)=\sum_{j=1}^{m} \bar{c}_{j}\left(v_{j}, f\right)
$$

so

$$
(u,(q+A) u)=(u, f)
$$

Since $A \geqslant 0$, we obtain

$$
(u, \varepsilon u)\left(\alpha^{2}-\beta^{2}\right)+(u, \sigma u) \alpha \leqslant \operatorname{real}(u, f)
$$

and

$$
(u, \varepsilon u) 2 \alpha \beta+(u, \sigma u) \beta=\operatorname{imag}(u, f) .
$$


Because $\varepsilon, \sigma$ and $\alpha$ are all positive, $\beta$ and imag $(u, f)$ must have the same sign. Multiply (11) by $\alpha,(12)$ by $\beta$ and add to obtain

$$
(u, \varepsilon u) \alpha\left(\alpha^{2}+\beta^{2}\right)+(u, \sigma u)\left(\alpha^{2}+\beta^{2}\right) \leqslant \alpha \operatorname{real}(u, f)+\beta \operatorname{imag}(u, f) .
$$

Hence,

$$
|s|^{2}[(u, \varepsilon u) \alpha+(u, \sigma u)] \leqslant 2|s||(u, f)|
$$

It now follows that

$$
|s|^{2}\|u\|^{2}\left[\varepsilon_{*} \alpha+\sigma_{*}\right] \leqslant 2|s|\|u\|\|f\|,
$$

from which we obtain

$$
\|u\| \leqslant 2|s|^{-1}\left(\varepsilon_{*} \alpha+\sigma_{*}\right)^{-1}\|f\| .
$$

For each $m$ we may construct in this way a solution of (6), which we now denote by $u_{m}$. The sequence $\left\{u_{m}\right\}$ is bounded, so we can select a subsequence $\left\{u_{m_{l}}\right\}$ such that $u_{m_{i}} \rightarrow u$ weakly as $i \rightarrow \infty$. Consider the equation

$$
\left(v_{j},(q+A) u_{m_{l}}\right)=\left(v_{j}, f\right) \text { for fixed } j \leqslant m_{i} .
$$

Then

$$
\left(v_{\jmath}, q u_{m_{l}}\right)+\left(A v_{j}, u_{m_{l}}\right)=\left(v_{j}, f\right) .
$$

In the limit as $i \rightarrow \infty$,

$$
\left(v_{\jmath}, q u\right)+\left(A v_{\jmath}, u\right)=\left(v_{\jmath}, f\right) .
$$

Since this holds for all $j$, we have

$$
(v, q u)+(A v, u)=(v, f) \text { for all } v \in D(A),
$$

so the form

$$
v \rightarrow(A v, u)
$$

is continuous on $D(A)$ in the $L^{2}$ topology. Thus, $u \in D\left(A^{*}\right)=D(A)$, and

$$
(v,(q+A) u)=(v, f) \text {. }
$$

This shows that $u$ is a solution of

$$
(q+A) u=f
$$

Clearly,

$$
\|u\| \leqslant 2|s|^{-1}\left(\varepsilon_{*} \alpha+\sigma_{*}\right)^{-1}\|f\| .
$$

Since $(q+A)$ is an entire function of $s,(q+A)^{-1}$ will be holomorphic wherever it exists: in particular, $(q+A)^{-1}$ will be holomorphic for $\alpha>0$. Thus, if $f$ is holomorphic for $\alpha>\Theta \geqslant 0$, so too will be $u$.

A corollary of this theorem is the existence of a unique solution of (2), provided that the source current density $K$ is sufficiently well behaved to permit the inversion of the Laplace transform. 
COROLlaRY. Suppose that the $L^{2}$-valued function $K$ has the following properties

(1) $K$ has support $0 \leqslant t<\infty$;

(2) $K$ is infinitely differentiable with respect to $t$;

(3) $K$ is Laplace transformable for real $s>0$.

Then the equation

$$
\mu \varepsilon \partial^{2} E / \partial t^{2}+\mu \sigma \partial E / \partial t+\nabla \times(\nabla \times E)=-\mu \partial K / \partial t
$$

has a unique solution with the same properties.

REMARK. At the cost of quite considerable notational complexity, one could use the same argument to prove existence and uniqueness for sources $K$ which are distributions in the $t$ variable, provided one also invokes results on distributional Laplace transforms. We attempted to write these results down, but found that the additional machinery merely obscured the essential simplicity of our arguments, and so we decided to omit this refinement.

It is worth noting the class of sources for which the spatial distribution is fixed and only the amplitude changes with time. An example is a fixed conducting loop driven by a current source. For such a source, $K$ is separable,

$$
K(t, x)=L(t) M(x),
$$

and will certainly satisfy the conditions of the lemma if $M$ is an $L^{2}$ function and $L$ is a smooth, Laplace transformable function with support $0 \leqslant t<\infty$.

\section{Quasi-static limit}

The quasi-static limit represents the physically interesting situation in which a wave process degenerates into a diffusion process. More precisely, the limit applies to observations in a bounded region of space long after wave fronts from the sources have passed. Mathematically, the limit is fairly subtle, because it corresponds to the vanishing of the coefficient of the second-order time derivative in the wave equation.

In order to examine the limit as $\varepsilon \rightarrow 0$, we replace $\varepsilon$ by $\lambda \varepsilon$ and allow $\lambda$ to approach zero while $\varepsilon$ is held fixed. Let $E_{\lambda}$ and $e_{\lambda}$ denote the corresponding solutions of (2) and (3). We observe that:

$$
e_{\lambda}-e_{0}=\left(q_{\lambda}+A\right)^{-1} \lambda \varepsilon s^{2}\left(q_{0}+A\right)^{-1}(\mu s k)
$$

so

$$
\left\|e_{\lambda}-e_{0}\right\| \leqslant 4 \lambda \varepsilon^{*} \sigma_{*}^{-2} \mu|s|\|k\|
$$


where $\varepsilon^{*}$ is the maximum value of $\varepsilon$. Clearly, $e_{\lambda} \rightarrow e_{0}$ pointwise in $s$ as $\lambda \rightarrow 0$. Since

$$
\left(E_{\lambda}-E_{0}\right)(t)=\frac{1}{2 \pi i} \int_{C} e^{s t}\left(e_{\lambda}-e_{0}\right)(s),
$$

where $C$ is any vertical contour in the right half plane, we have

$$
\left\|E_{\lambda}-E_{0}\right\| \leqslant \frac{1}{2 \pi} 4 \lambda \varepsilon^{*} \sigma_{*}^{-2} \mu \int_{C}\left|e^{s t}\right||s|\|k(s)\| .
$$

Since $\|k\|$ decreases faster than any power of $|s|$, the appearance of the extra power of $s$ in the integrand does not affect the convergence of the integral, so we find that $E_{\lambda} \rightarrow E_{0}$ in $L^{2}$ as $\lambda \rightarrow 0$.

The results of this and the preceding section are not surprising on physical grounds. From a practical viewpoint they imply that a numerical computation of a solution to (2) for $\varepsilon=0$ should be in good agreement with the true solution under the conditions prevailing in the geophysical context.

On the other hand, it is unusual in an elliptic problem to obtain solutions which are only $L^{2}$, especially when the source term can be made as smooth as one likes. The 'bad' behaviour of the solutions is, as we shall see in the next section, due entirely to the assumption that $\varepsilon, \sigma$ are only piecewise constant. (If $\varepsilon, \sigma$ are smooth then it is straightforward to show that the smoothness of the solution is determined entirely by that of the source.) Knowing a priori precisely how bad the solutions are is an essential input to formulating a finite-element numerical scheme for solving (2).

\section{Regularity}

We shall establish four regularity results

(1) away from interfaces, all fields are smooth if the source $K$ is smooth;

(2) the transverse component, $e_{T}$, lies in $H^{2}$, and so is certainly continuous;

(3) the longitudinal component, $e_{L}$, lies in $L^{2}$, but cannot lie in $H^{1}$;

(4) $n \times e$ and $n \cdot(\sigma e)$ are continuous across any interface $\Gamma$ in the sense of distributions in $H^{-1 / 2}(\Gamma)$.

These results are not necessarily optimal, but they do show that the electromagnetic fields cut across the boundaries of the conventional Sobolev spaces. Nonetheless, result (4) is all that is needed to establish the numerical analysis of Maxwell's equations. Because of their technical nature, we present the proofs of these facts as four lemmas. 
We shall obtain the regularity results for $e$ in the Laplace domain. The first result is the standard elliptic regularity result away from interfaces.

Lemma 2. Let $q_{i}$ denote the value of $q$ on $\Omega_{i}$. If

$$
q_{1} k+\nabla \nabla \cdot k \in H_{\mathrm{loc}}^{r}\left(\Omega_{1}\right) \text {, }
$$

then $e \in H_{\mathrm{loc}}^{r+2}\left(\Omega_{i}\right)$. In particular, if $k$ is smooth in $\Omega_{l}$, then so too is $e$.

Proof. Let $\Omega$ be any open subset of $\Omega_{i}$, and choose a smooth function $\phi$ in $D\left(\Omega_{1}\right)$ which is equal to one on $\Omega$. Then

$$
\begin{aligned}
\nabla \times(\nabla \times(\phi e))= & \phi \nabla \times(\nabla \times e)+\nabla \phi \times(\nabla \times e)+e \cdot \nabla \nabla \phi+\left(\nabla^{2} \phi\right) e \\
& -\nabla \phi \nabla \cdot e+(\nabla \phi \cdot \nabla) e .
\end{aligned}
$$

Although this expression contains mixed derivatives, such as $(\nabla \phi \cdot \nabla) e$ over which we have no control, if we choose $\psi$ in $D(\Omega)$ then

$$
(\psi,(q+\nabla \times \nabla \times)(\phi e))=-\mu s(\psi, k) .
$$

In particular, if $\psi=\nabla \chi$, with $\chi \in D(\Omega)$, then

$$
(\chi, \nabla \cdot(q e))=-\mu s(x, \nabla \cdot k),
$$

from which it follows that

$$
\nabla \cdot e=-\mu s q_{t}^{-1} \nabla \cdot k \quad \text { in } \Omega .
$$

Hence,

$$
\begin{aligned}
\left(-\nabla^{2}+q_{\imath}\right) e & =\nabla \times(\nabla \times e)-\nabla \nabla \cdot e+q_{\imath} e \\
& =-\mu s k-\left(\mu s / q_{\imath}\right) \nabla \nabla \cdot e \\
& =-\mu s\left(k+q_{l}^{-1} \nabla \nabla \cdot k\right) \text { in } \Omega .
\end{aligned}
$$

If $k+q_{t}^{-1} \nabla \nabla \cdot k \in H_{\text {loc }}^{r}\left(\Omega_{t}\right)$, then $e \in H_{\text {loc }}^{r+2}(\Omega)$ by elliptic regularity (Lions and Magenes [2, page 125]).

In order to examine regularity near the boundaries, we shall split $e$ into its longitudinal and transverse parts,

$$
e=e_{T}+e_{L}
$$

where

$$
e_{T}=P_{T} e \text { and } e_{L}=P_{L} e
$$

LEMma 3. The transverse component, $e_{T}$, of the solution of (3) lies in $H^{2}$. Consequently, $e_{T}$ is continuous.

Proof. We know that $e_{T} \in L^{2}$, that $\nabla \cdot e_{T}=0$, and that $\nabla \times e_{T}=\nabla \times e \in$ $L^{2}$, so from Lemma 1 it follows that $e_{T} \in H^{1}$. But we also know that $\nabla \times e_{T} \in$ $H^{1}$, so again using Lemma 1 we find that $e_{T} \in H^{2}$. It is a standard embedding theorem that every function in $H^{2}$ is also continuous. 
The longitudinal component $e_{L}$ certainly lies in $L^{2}$, but cannot lie in $H^{1}$. Indeed, the classical argument shows that $e$ must be discontinuous, which implies that $e_{L}$ is discontinuous because Lemma 3 shows $e_{T}$ to be continuous. Hence, $\nabla \cdot e_{L}$ cannot lie in $L^{2}$. Lemma 1 then shows that $e_{L}$ cannot lie in $H^{1}$. Whether $e_{L}$ lies in $H^{r}$ for some $r$ in the range $0<r<1$ is an open question.

Following closely Duvant and Lions [1], we introduce the spaces

$$
\begin{aligned}
& H(\operatorname{curl}, \Omega)=\left\{\phi \mid \phi \in L^{2}(\Omega), \nabla \times \phi \in L^{2}(\Omega)\right\} \\
& H(\operatorname{div}, \Omega)=\left\{\phi \mid \phi \in L^{2}(\Omega), \nabla \cdot \phi \in L^{2}(\Omega)\right\}
\end{aligned}
$$

with the graph norms

$$
\left(\|\phi\|^{2}+\|\nabla \times \phi\|^{2}\right)^{1 / 2} \text { and }\left(\|\phi\|^{2}+\|\nabla \cdot \phi\|^{2}\right)^{1 / 2},
$$

respectively. Then $C_{K}^{1}(\bar{\Omega})$ is dense in both these spaces and the maps

$$
u \rightarrow n \times\left. u\right|_{\Gamma},\left.\quad u \rightarrow n \cdot u\right|_{\Gamma}
$$

from $C_{K}^{1}(\Omega)$ to $C^{1}(\partial \Omega)$ can be extended by continuity to mappings, again denoted by

$$
u \rightarrow n \times u \quad u \rightarrow n \cdot u,
$$

which are linear and continuous from

$$
H(\operatorname{curl}, \Omega) \rightarrow H^{-1 / 2}(\Gamma) \text { and } H(\operatorname{div}, \Omega) \rightarrow H^{-1 / 2}(\Gamma),
$$

respectively. Furthermore, for all $\phi \in H^{1 / 2}(\Gamma)$ and $\Phi \in H^{1}(\Omega)$ such that $\left.\Phi\right|_{\Gamma}=$ $\phi$,

$$
\begin{array}{cc}
\langle\phi, n \times u\rangle=\int_{\Omega}(\Phi \cdot \nabla \times u-u \cdot \nabla \times \Phi), & u \in H(\operatorname{curl}, \Omega), \\
\langle\phi, n \cdot u\rangle=\int_{\Omega}(\Phi \nabla \cdot u-u \cdot \nabla \Phi), & u \in H(\operatorname{div}, \Omega),
\end{array}
$$

where it should be remembered that $\phi$ and $\Phi$ are understood to be vector-valued in (13), but scalar-valued in (14).

Now suppose that $\Omega$ is a region which spans a section, $\Gamma$, of the interface between $\Omega_{+}$and $\Omega_{-}$. Let

$$
\Delta_{ \pm}=\Omega \cap \Omega_{ \pm}
$$

and let

$$
\Gamma=\bar{\Delta}_{+} \cap \bar{\Delta}_{-} .
$$

Choose any $\phi \in H^{1 / 2}(\Gamma)$ and let $\Phi_{ \pm}$denote liftings of $\phi$ to $H^{1}\left(\Delta_{ \pm}\right)$,

$$
\left.\Phi_{ \pm}\right|_{\Gamma}=\phi \text {. }
$$

Without loss of generality we may assume that

$$
\text { support } \Phi_{ \pm} \subset \Delta_{ \pm} \text {. }
$$


Indeed, let $\psi \in D(\Omega)$ with $\psi=1$ in a neighbourhood of $\Gamma$. Then $\psi \Phi_{ \pm}$also lifts $\phi$ and has the assumed properties.

Lemma 4. Suppose $e \in L^{2}$ and $\nabla \times e=0$ in $\Omega$. Let $n \times e_{ \pm}$denote the tangential trace of $n \times e$ on $\Gamma$ from $\Omega_{ \pm}$. Then

$$
n \times e_{+}=n \times e_{-}
$$

in the sense of equality of distributions in $H^{-1 / 2}(\Gamma)$.

Proof. For any $\phi$ in $H^{1 / 2}(\Gamma)$, it follows from (13) that

$$
\left\langle\phi, n \times e_{ \pm}\right\rangle= \pm \int_{\Delta_{ \pm}}\left(\Phi_{ \pm} \cdot \nabla \times e-e \cdot \nabla \times \Phi_{ \pm}\right)
$$

so

$$
\left\langle\phi, n \times e_{ \pm}\right\rangle=\mp \int_{\Delta_{ \pm}} e \cdot \nabla \times \Phi_{ \pm} \cdot
$$

Thus,

$$
\left\langle\phi, n \times e_{+}-n \times e_{-}\right\rangle=-\int_{\Omega} e \cdot \nabla \times \Phi
$$

where

$$
\Phi(x)= \begin{cases}\Phi_{+}(x), & x \in \Delta_{+}, \\ \Phi_{-}(x), & x \in \Delta_{-} .\end{cases}
$$

Since support $\Phi \subset \Omega$, integration by parts gives

$$
\left\langle\phi, n \times e_{+}-n \times e_{-}\right\rangle=0 \text { for all } \phi \in H^{1 / 2}(\Gamma) .
$$

We now turn to the normal components of $\sigma e$ and focus upon a region $\Omega$ where $\nabla \cdot k=0$. It then follows from (3) that

$$
\nabla \cdot(\sigma e)=0 \text { in } \Omega \text {. }
$$

Lemma 5. Suppose $f \in L^{2}$ and $\nabla \cdot f=0$ in $\Omega$. Let $n \cdot f_{ \pm}$denote the normal trace of $n \cdot f$ on $\Gamma$ from $\Omega_{ \pm}$. Then

$$
n \cdot f_{+}=n \cdot f_{-}
$$

in the sense of equality of distributions in $H^{-1 / 2}(\Gamma)$.

Proof. For any $\phi$ in $H^{1 / 2}(\Gamma)$, it follows that

$$
\left\langle\phi, n \cdot f_{ \pm}\right\rangle= \pm \int_{\Delta_{ \pm}}\left(\Phi_{ \pm} \nabla \cdot f-f \cdot \nabla \Phi_{ \pm}\right),
$$


so

$$
\left\langle\phi, n \cdot f_{ \pm}\right\rangle=\mp \int_{\Delta_{ \pm}} f \cdot \nabla \Phi_{ \pm} .
$$

Thus,

$$
\left\langle\phi, n \cdot f_{+}-n \cdot f_{-}\right\rangle=-\int_{\Omega} f \cdot \nabla \Phi
$$

where

$$
\Phi(x)= \begin{cases}\Phi_{+}(x), & x \in \Delta_{+}, \\ \Phi_{-}(x), & x \in \Delta_{-} .\end{cases}
$$

Since support $\Phi \subset \Omega$, integration by parts gives

$$
\left\langle\phi, n \cdot f_{+}-n \cdot f_{-}\right\rangle=0 \text { for all } \phi \in H^{1 / 2}(\Gamma) .
$$

We now observe that $e_{L}$ and $\sigma e$ satisfy the conditions of Lemma 4 and Lemma 5 , respectively, so $n \times e_{L}$ and $n \cdot(\sigma e)$ are continuous across $\Gamma$ in the sense of distributions in $H^{-1 / 2}(\Gamma)$.

\section{Acknowledgement}

Both authors would like to thank the Department of Economic Geology at the University of Adelaide for hospitality while this research was carried out.

\section{References}

[1] G. Duvaut and J. L. Lions, Inequalities in mechanics and physics (Springer, Berlin, New York 1976).

[2] J. L. Lions and E. Magenes, Non-homogeneous boundary value problems and applications I (Grundlehren math. Wiss. 181) (Springer, Berlin, New York 1972).

[3] D. M. O'Brien and R. S. Smith, "Transient electromagnetic response of a layered conducting medium at asymptotically late times", J. Austral. Math. Soc. Ser. B 27 (1985), 1-30.

[4] J. R. Wait, Geoelectromagnetism (Academic Press, New York 1981). 